Transactions of the SDPS:

Journal of Integrated Design and Process Science

16 (3), 2012, 133-148

DOI 10.3233/jid-2012-0017

http://www.sdpsnet.org

\title{
On the Transdisciplinary Field of Humor Research
}

\author{
Julia M. Taylor and Victor Raskin ${ }^{*}$ \\ CIT \& CERIAS and LING \& CERIAS, Purdue University, West Lafayette, Indiana, USA
}

\begin{abstract}
Based on formal theories of humor and a computational semantic technology for information and text meaning analysis, computers could be programmed to detect and generate humor and determine the patterns of individual humor preferences both for the joke tellers or posters and for the people that comment on jokes. These patterns do not only reveal information on a person's sensitivities to various spheres of life, which, in turn, aggregated, when necessary, over any number of individuals, can be used for targeted advertising, focused marketing, and possibly political campaigns and definitely for cyber security, but also provide a solid cognitive and emotional foundation for friendlier human computer interaction. The paper introduces the rationale and theory for rigorous study of humor that provides a foundation for the outlined research.
\end{abstract}

Keywords: humor research, humor theory, linguistics of humor, Ontological Semantic Theory of Humor (OSTH), computational humor, social computing, individual humor preferences

\section{What Is a Transdisciplinary Theory of Humor}

\subsection{From the disciplinary to inter- to transdiciplinary}

At the last meeting of the Society for the Design and Processes Study in Berlin in June 2012, the central notion that emerged in several high-powered discussions, especially those on the image, substance, and future of the Society, as the next generation was taking over the leadership, was that of transdisciplinarity. The Society has always attracted interdisciplinary contributions. Typically, in interdisciplinary research, a scholar in one field tries to utilize the findings in a different, often adjacent field to advance his or her own field. Sometimes a scholar from that other field participates; often not. What usually characterizes such research is the lack of equality between the two fields: the research follows the methodology and often format of the one field (target field) and applies some elements of the other (source field) to augment them (Figure 1).

Multidisciplinarity is interdisciplinarity times $\mathrm{x}$ as far as the source fields are concerned. The target field is still single, and the asymmetry of interdisciplinarity remains. To date, Ruch et al. (Ruch et al., 1993) remains a unique example of true multidisciplinarity in humor research as the target and

\footnotetext{
Corresponding author. E-mail: vraskin@purdue.edu
} 
psychology of humor and linguistics of humor as the two source field, themselves interdisciplinary fields, with humor research, again, as target fields and psychology and linguistics, respectively, as the source fields.

\begin{tabular}{|ll|}
\hline Source Field & Target Field \\
\hline Data & Data \\
Theories & Theories \\
Methods & Methods \\
& \\
\hline Problems & Problems \\
\hline
\end{tabular}

Fig. 1. Application of a source field to a target field.

Transdisciplinarity removes much of this asymmetry: a transdisciplinary theory of a field is a true synthesis of the theories of a number $(N)$ of contributing fields, including the specific theory of that first field. This washes out the privileged position of a target field and replaces it with a set of $N+1$ source fields. The next section suggests what a transdisciplinary theory looks like.

\subsection{What is a proper theory}

Many scholars proceed to do major research without defining any theory or using the term. Following Chomsky (Chomsky, 1965), we firmly believe that a theory underlies any piece of research or, even mote universally, any statement. But when it is not stated explicitly it remains implicit, unseen and open for consideration and discussion. So this answers the question, who needs a major theory of humor? It is an illegal question that should be replaced with this correct one, who needs to see clearly and to be able to explicate and to defend the theory underlying their research? While many theoretically immature fields will answer that with the timid to arrogant, this is how it is done, a new developed field should take full advantage of transparency and explicitness.

The next question, what does the major theory of a field do? is perfectly legitimate. A major theory:

- ranges over the entire domain of the field

- takes responsibility for each phenomenon, rule, etc.

- determines the associated methodology, or the toolbox

- defines each specific work in terms of its;
o format
o premises
0 goals
o criteria of success

The major theory does all of that in pursuit of its major goal that can be summarized as determining the set of necessary and sufficient conditions for all major questions that the theory attempts to provide the answers in its body. With the body as its first, central, and often the only explicit component, a theory contains the following parts:

- Body of the Theory: set of explanatory and predictive statement about purview

- Purview: the phenomena that the theory takes on itself to deal with—or what it is the theory of

- Premises: the implicit axiomatic statements that the theory takes for granted-these are not stated clearly by many theories and cause most misunderstanding

- Goals: the final results of the successful formulation of a theory; 
- Methods of falsification: the clearly stated hypothetical situation which would prove the theory wrong, a counterexample-we follow here Karl Popper's (Popper, 1972) view that a hypothesis that is unfalsifiable in principle is not only not a theory but is actually a faith.

- Method of justification/evaluation: a set of statements on how to check the veracity of the body statements and, wherever possible, on how to compare the theory to its competition, if any.

A theory is not open to misinterpretation only when characterized by all of these features: it is

- adequate, if it provides an accurate account of all the phenomena in its purview;

- effective, if it comes with a methodology for its implementation;

- $\quad$ constructive, if that implementation can be completed in finite time;

- decidable, if there is an algorithm for its implementation in principle;

- computable, if this algorithm can actually be demonstrated,

- explicit, if it is fully aware of all of its components and provides a full account of each of them;

- formal, if it submits itself to logical rules, whether it does or does not use a specific formalismconfusing formality with formalism is one of the worst and unfortunately common offenses in discussing a formal theory.

So, a proper theory is to be seen as a part of the theory-methodology-description triple, where:

- description is what each phenomenon in the purview of the theory requires to be accounted for as;

- methodology is the set of ways to produce each description, and

- theory determines the format of each description and shaping and steering the appropriate methodology.

\subsection{What is a transdisciplinary theory of humor}

A proper theory of humor should then have all the components and features of a proper theory and have a humor-specific methodology for producing humor descriptions and representations.

- Body: all statements containing:

0 sets of necessary and sufficient conditions for what is funny;

o classes of humor and features constituting them;

0 formats of descriptions

- Proper purview: humor (all forms);

- Premises-mostly that:

o the phenomenon of humor is based on recognizable or predictable patterns;

0 these patterns can be described with a finite and/or recursive set of statements in the body of the theory;

o the theory comes with an algorithmic or superalgorithmic methodology;

o the result of applying this methodology to humor material, within the parameters of the theory, will result in a finite and/or algorithmically extensible set of descriptions and predictions;

o humans, in a reliably designed experiment, will recognize these descriptions and predictions as reflecting accurately their comprehension of humor, with an acceptable rate of agreement;

- Goals-mostly to:

o account for how each act and kind of humor works;

o for such accounts to add up to understanding humor the way people do:

o to go beyond that to a full explanation, the way people don't;

- Falsification: to produce a counterexample to any main statement in the body;

- Justification: to design and conduct experiments confirming the validity of the theory.

What makes a humor theory transdisciplinary is the establishment of a separate and distinct discipline of humor research, not as an application of a source filled with its own theory. A pioneering attempt to do 
so was undertaken, somewhat abortively, in Apte (Apte, 1988). Having termed it 'Humorology,' Apte (Apte, 1988: 9) defined it as "the study of the causes, nature- that is, form and substance-and functions of the phenomenon labeled humor"-see also Carrell (Carrell, 2008). This goal has been in the forefront of humor research since, and while very few scholars actively and explicitly pursue it, it is implicitly accepted that everything that is done in the field should be seen as leading in this direction.

Now, Apte's humorology looked suspiciously like anthropology, the discipline in which he trained in Chomskian linguistics, meaning largely syntax, which he repudiated, made his distinguished career. To some humor researchers from largely atheoretical disciplines, the view of theory proposed here looks suspiciously like linguistics, and its main proponent has been charged with a barbarous act of "linguistic imperialism" (Kuipers, 2002). In his defense, it can be argued that linguistics is the most theoretically advanced source field for humor research, and that should explain the fact that the view of theory proper may come from linguistics but without dragging any specifically linguistic content with it. Nevertheless, a truly transdisciplinary theory of humor should be indeed a true synthesis of the contributing theories, and a synthesis that is mightier than each of its contributors and clearly distinct from them. Realizing what a proper theory should look like is a major step towards true transdisciplinarity but has yet to be achieved.

\section{Humor Research}

\subsection{Why study humor?}

We hope this not too late to pose and answer this question here rather than risking to lose a number of serious potential readers on page one. One can, of course, use the famous Everest argument: "because it is there!” There, it is indeed, and quire persistently, at that. It is a universal human faculty, manifesting itself variously in different cultures but sharing its basic traits and preserving them through different languages. The "Anglo-Saxon" societies may place a high premium on using humor in public addresses while the German, Arab, and Japanese societies prefer to limit humor to personal or even intimate circle but the humor used may be identical, and the need to use is there in the same way.

Humor has been shown to be an important part of human communication ability. If a language learner acquires the ability, first, to detect and appreciate humor in the foreign language and then, later, make jokes in it, this can serve as a high measure of the learner's acquired competence (Vega, 1989; Deneire 1995). An important tool of social bonding, the sense of humor is an important indicator of adaptability, and to the extent it can be developed, it offers one way to improve one's social, organizational, and leadership skills, at least in Western societies. Research shows that mental health can also be determined by the study of how patients respond to humor. While numerous health-and-humor seminars are not always ranked high by humor researchers (Martin, 2008), there are some indications that laughter may have beneficial effects. Practically, any one of the source fields addressing humor research points out to an important role humor plays in personal, social, professional, and political lives of people.

Also, as we will show below as well, it is beginning to be much better understood how important humor is for normal, full-fledged human communication when intelligent agents are designed to be the only companion and interlocutors for people who live alone and do not go out much. It is also important to detect humor and not to confuse it with bona-fide information in all forms of computational text and information processing. So yes, it is indeed there, and we'd better know where, when, and especially what it is.

The idea that humor can be analyzed, formalized and computed still surprises many scholars, especially those from the STEM disciplines. Like other non-discrete, natural or even human phenomena, humor is patterned and often formulaic. Moreover, it is no more foreign to humans than logical reasoning or game theory, and could be arguably as needed for human friendly computing. Besides explicitly templated jokes, such as Knock-Knock or Light Bulb ones, there are many other similarly organized 
jokes. Where discrete features with fillers are identified, there is a possibility for formalization. For verbal jokes, it is related to the formalization of linguistics in the $20^{\text {th }}$ century, which started in phonology and then extended to syntax and, later, to semantics, which is most relevant to jokes.

\subsection{How has humor been researched}

In general, work on humor theories has a long history, and, to this day, the true nature of humor is still being debated (Raskin, 1985; Morreall, 1983; Oring, 1992; Ruch, 1998; Davies, 1990; Attardo, 1994). There is no universally accepted theory of humor that explains "what is funny, why it is funny, how it is funny, when it is funny, and to whom it is funny” (Raskin, 1985: 5). Most humor models and theories can be divided into three classes. According to the most common classification (Morreall, 1983; Raskin, 1985; Ritchie, 2004), humor theories can be based on: incongruity, relief/release or hostility/superiority/ aggression/malice/disparagement. Attardo (Attardo, 1994) divides humor theories into three similar classes, but gives the classes different names, as shown in Figure 2.

\begin{tabular}{lll}
\hline \hline Cognitive & Social & Psychoanalytical \\
\hline incongruity & hostility & release \\
\hline contrast & aggression & sublimation \\
& superiority & liberation \\
& triumph & economy \\
& derision & \\
& disparagement & \\
\hline \hline
\end{tabular}

Fig. 2. Types of Humor Theories (Attardo, 1994: 47).

Incongruity-based theories suggest that humor arises from something that violates an expectation. Many supporters of incongruity in humor have followed Kant in emphasizing the importance of surprise in a joke (Kant, 1790). Superiority theories are based on the observation that people laugh at other people's infirmities, especially if they are enemies (Rapp, 1951; Suls, 1976; Grunner, 1978). This class of theories of humor goes back to the philosophers of Ancient Greece and Rome, many of whom maintained that people laugh at the misfortunes of others for joy that they do not share them (Raskin, 1985; Attardo, 1994). Release/relief theories explain the link between humor and laughter. The principle for the release-based theories is that laughter "provides relief for mental, nervous and psychic energy, and this ensures homeostasis after a struggle, tension, and strain” (Raskin, 1985: 38). "The most influential proponent of the release theory is certainly Freud" (Attardo, 1994: 50).

These classes of theories can be also roughly summarized as follows (Ritchie, 2000): “...cognitive/incongruity approaches concentrate on the humorous stimulus, social/hostility approaches consider the interpersonal effects, and psychoanalytical/relief proposals emphasize the audience's reaction." Each discipline's contribution to its working definition of humor can also form a basis for computational recognition of humor, as needed for this paper. From the Artificial Intelligence perspective, the theories, when they exist, take a bottom-up approach and are limited in applicability to a narrower humor form (Ritchie, 2000; Ritchie, 2004). From the psychological perspective, it has been known since Freud (Freud, 1905), that the human humor competence includes the realization that some jokes are unsuitable for certain individuals with specific sensitivities (cf. Veatch, 1998).

\section{Linguistic Theory of Humor}

The linguistic theories of humor (Raskin, 1985; Attardo \& Raskin, 1991; cf. Attardo, 1994), whose components can be roughly defined by adding 'verbal' or 'textual' before each occurrence of 'humor 
have finally reached a level of formal representation that is adaptable for the computation for any humorous text (Raskin et al, 2009a; Raskin et al, 2009b; Taylor, 2011; Hempelmann, 2011; Raskin, 2011a).

\subsection{Script-based Semantic Theory of Humor (SSTH)}

The best known and most used, even if outdated, linguistic theory of humor is the Script-based Semantic Theory of Humor (SSTH: Raskin, 1985). It was the first-ever use of a formal theory, even though offered without much formalism and thus palatably for an audience that was addressing humor primarily from the perches of the humanities (mostly, literary studies) and social sciences (mostly, psychology but, increasingly, sociology). As a result, the theory impressed much more people and much more greatly than it should have, and the clearly stated point that the theory's applicability was contingent on the development of a meaning-based computational system of natural language processing, then over a decade away, was lost on its non-NLP-expert readership almost entirely.

According to the theory, there are two conditions for a text to be humorous:

- A text has to be compatible, fully or in part, with two different scripts.

- The two scripts with which the text is compatible are opposite, and with which it must overlap fully or partially.

The compatibility of the text with two scripts is the necessary condition for humor; the oppositeness of the scripts is the sufficient condition. A script "is an enriched, structured chunk of semantic information, associated with word meaning and evoked by specific words. The script is also a cognitive structure internalized by the native speaker, and it represents the native speaker's knowledge of a small part of the world. [...] Formally or technically, every script is a graph with lexical nodes and semantic links between the nodes" (Raskin, 1985: 81). Scripts were further developed in Ontological Semantics (Raskin et al., 2003; Nirenburg and Raskin, 2004).

The scripts can be linguistic, general knowledge, restricted, or individual. Linguistic scripts are known to any "average," "standard" native speaker (adult, reasonably educated, mainstream culture, etc). General knowledge scripts, such as crossing the street or going to a store, are known to a large number of people and are not affected by their use of language. Restricted knowledge scripts are known to a smaller number of people and are not affected by their use of language either. Individual scripts are "owned" by one person: an example of an individual script would be a child's memory of her first swim. Basing the theory on Ontological Semantic Technology (OST_see also below)) allows a crisper definition of the necessary and sufficient conditions for verbal humor (Raskin et al., 2009a; Raskin et al., 2009b).

\subsection{General Theory of Verbal Humor (GTVH)}

The General Theory of Verbal Humor (Attardo \& Raskin, 1991), proposed as an attempt to improve and enrich SSTH, is a linguistics-centered multidisciplinary theory of humor that is still built upon the notion of script overlap and script oppositeness. The theory describes jokes in terms of six knowledge resources (Attardo, 1994):

- Script Opposition (SO): deals with script overlap and oppositeness presented in Script-based Semantic Theory of Humor (SSTH).

- Logical Mechanism (LM): accounts for the way in which the two scripts in the joke are brought together.

- Situation (SI): the "props" of the joke, the textual materials evoked by the scripts of the joke that are not necessarily funny.

- $\quad$ Target (TA): any individual or group from whom humorous behavior is expected.

- Narrative Strategy (NS): the rhetorical structure of the text; that is the "genre" of the joke, such as the riddle, 1-2-3 structure, question and answer, etc. 
- Language (LA): the actual lexical, syntactic, phonological, etc., choices at the linguistic level that instantiate all the other choices; language is responsible for the position of the punchline.

According to the General Theory of Verbal Humor, each joke can be viewed as a 6-element vector, specifying the instantiation of each parameter:

Joke: $\{L A, S I, N S, T A, L M, S O\}$

The only element that is optional in a joke description is the Target (TA), but it is routinely used in the political and social humor, which is of relevance for this paper. Equally important is the Situation (SI) parameter, as it anchors all the opinions, attitudes and sensitivity in the real events that the jokes mention or refer to.

\subsection{GTVH justification}

The theory was, for the most part, validated in a large scale psychological experiment (Ruch et al., 1993), and this attempt at justifying a proper theory of humor has remained, unfortunately unique in the field of humor research. What the experiment, which was a serendipitous add-on of a few dozen questions to a much larger questionnaire in a massive special-education experiment, with a few thousand middleschool students in Central Indiana, establishing whether using humor in the classroom was effective, attempted to justify was the hierarchy of GTVH's KRs. As shown above, SO is the deepest-seated level while LA is the most superficial one.

The experiment-or rather, the GTVH sub-experiment—has this initially plausible hypothesis: if two jokes differ at a deeper KR level the subjects will find it harder to see the similarities than if they differ at a more superficial level. So, going to the extremes, if two jokes share all the 5 underlying KRs and differ only in the wording of the jokes, the similarities will be much more recognizable than if two jokes differ in their SO while sharing all the other, more shallow KRs. The sub-experiment questions were dispersed among the questions of the master experiment and masterfully camouflaged by Ruch's brans of superior design.

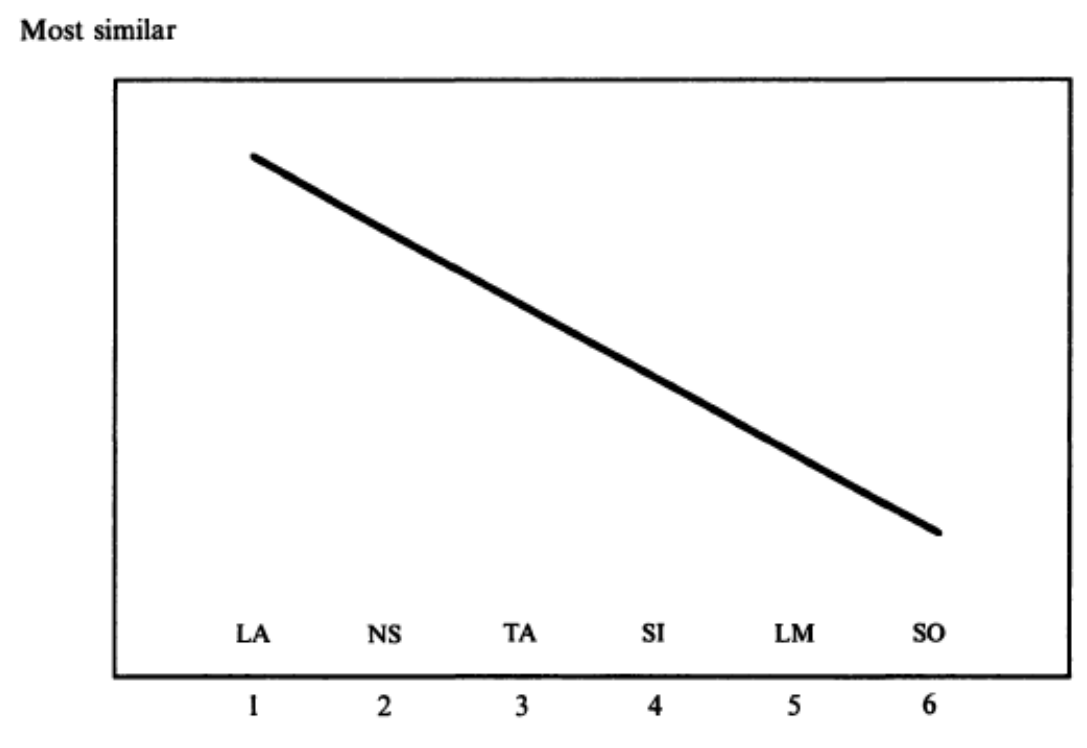

Fig. 3. Previsioned results.

Figure 3 shows the ideally previsioned results while Figure 4 shows the actual results of the experiment. The results are good for all but the LM KR, and a long discussion ensued among the proponents about the legitimacy of that component (Hempelmann, 2004; Hempelmann, 2009; 
Hempelmann, 2012; Hempelmann \& Attardo, 2011; Davies, 2011). GTVH sustained a heavy but, basically, misguided attack from Oring (Oring , 2011), rebuffed in Raskin (Raskin, 2011b).

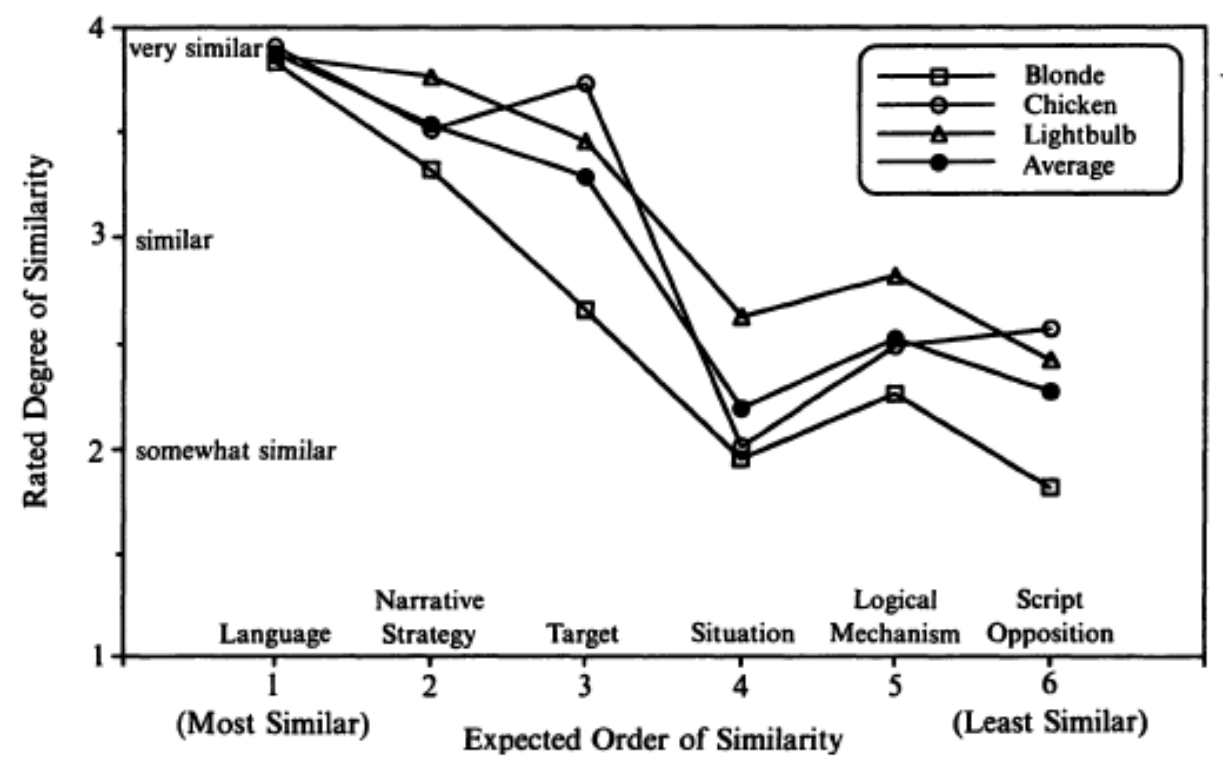

Fig. 4. Actual results.

\section{What Is Not in Humor Theory: The Audience Gap}

\subsection{Who gets it and who does not}

It was from inside SSTH that Carrell (Carrell, 1993; 1997a,b) first noticed that the influential theory had ignored the audience for humor entirely. Yet every performer knows that the same joke may be enjoyed by one audience and ignored or even booed down by another-possibly on the consecutive nights at the same Improv. The result of this gap is that it can only represent jokes as potential jokes: it will make you laugh given certain conditions.

The only issue that gained currency in traditional humor theory is Freud's statement that people would not find funny things that they have strong feelings about. A person who had a loss in the family would not appreciate jokes about death. One of the co-authors used to brag about his professional tolerance to any kind of humor but disdained the dead-baby jokes after his own only child had been borne.

Using scripts, one can then suggest that some scripts are not available for humor for certain individuals or groups. Ethnic humor became very rare as political correctness was setting in in the late 1980, and there were actual pickets of a movie about a struggling stand-up comedian who specialized in Polish jokes, and the picket line were set in the Polish neighborhoods of Chicago. Scripts unavailability for humor may be also explained by their unavailability, period. The male co-author realized that he stopped getting most Russian jokes online some 15 years after his emigration from Moscow: the TA and SI KRs often left him completely mystified. There were also new words and expressions that his former fellow countrymen had developed after he was no longer member of the same community-this can also be reduced to the unavailability of scripts: an unknown word or phrase will fail to evoke an appropriate script.

The scripts do provide a convenient explanation but, in fact, they only defer real explanation by one step: why is a certain script unavailable. This is where all kinds of life circumstances, birth, upbringing, 
education, careers, social life, etc., may all affect the outcome. So no theory of humor can be fully adequate without a full incorporation of psychology, sociology, anthropology, political science-and not even then.

\subsection{Individual and group humor analysis}

This paper is an extension of Taylor \& Raskin (Taylor \& Raskin, 2011), and this section explores the use of automatic computational humor analysis not only, and not even mainly, for humor human computer interaction, but, importantly, for determining the societal attitude and attention-or lack thereof-to the events and/or phenomena in the focus of jokes or witticisms, usually the TA and SI KRs. It also addresses the distribution of individual reactions over the spectrum of social, cultural and political opinions, as revealed in the individuals' sensitivities—or, again, lack thereof — to the jokes. Thus, humor analysis is not used here just for determining what type of jokes somebody likes, for the sake of telling jokes-it has much broader social implications and applications. It can also be used for social profiling, when and where legal, to expose and prevent social engineering, thus providing an additional defense against cyber crime and attacks.

Suppose a Facebook, Twitter or some other social media user posts a joke or improvises something humorous on a current event for everybody to see. The posting is followed by verbal comments, as well as like/dislike indications. Some of these are obviously positive, others are not. The question is, what can be learned from this material by an informed and unbiased human reader and how this information can be integrated, emulated, analyzed, and summarized by the computer. It should be emphasized here that we are not interested in words that explicitly indicate somebody's attitude towards a message, but rather what in particular in the message triggered a positive or negative response.

Thus, upon reading the following posting on the Political Humor open page on Facebook (October 16, 2010), a human reader understands that the joke is targeted at President George W. Bush, stereotyped as dumb, inarticulate and illiterate:

"Somebody threw a book at President Obama. If you're trying to scare a president by throwing a book at him, you're one president too late." —David Letterman

The 29 comments that followed the posting displayed the usual full range of Bush haters, Bush defenders, Obama haters and defenders, and comments on the joke itself as well as on the inappropriateness of the action. This is not at all surprising, as various polls would confirm. The polls, however, typically cluster their information in a certain way, whereas here, the explanation and justification for an individual opinion is available and can be used for other purposes.

The additional dimension elicited by a joke is the range of joke perception with regard to people's sensitivities to its target: people who categorically reject this joke as inappropriate reveal a strong sensitivity to the Office of President with the corresponding decorum; posters who applaud the joke may flaunt their lack of any such sensitivity, but reveal others, for instance, about the current or previous president; those who read the joke but don't indicate their appreciation of it signal an indifference to the topic, or at least don't display enough interest that would overcome their apathy.

The computer equipped with text understanding technology and capability for humor analysis, may easily collect and integrate numerous comments with all the additional material the posters bring into them for any individual joke, type of jokes or postings over a significant period of time. The understanding of a joke, especially in terms of its target and situation, is indeed essential for the task. Thus, the computer must understand that the joke below, again by David Letterman, targets the current president, as opposed to the past president in the joke above:

"People are kind of upset with British Petroleum CEO Tony Hayward. Over the weekend, he was out on his yacht. And when President Obama found out that Tony Hayward was on his yacht, he was so angry, he missed a putt."

Obviously, the computer can only "get” the joke if it can store and, unlike IBM's Watson (Ferrucci et al., 2010), also understand the element of world knowledge, no matter whether factually correct or not, 
that playing golf is just as elitist as sailing a yacht. Now, getting to this understanding is not easy for the computer just as it is not easy for a child or a foreigner, fluent in English but not in the American political and electoral ethos. At least three pieces of world knowledge should be available for understanding the joke, namely that:

- yachts are expensive and, hence, owned by or available to the rich;

- golf is historically associated with exclusive clubs that own and maintain the fields, and the high cost of the membership in these clubs has also traditionally limited the game to the wealthy;

- an American elected official must appeal to the majority of the electorate who are mostly not wealthy, and he or, more rarely, she should posture as the defender of the poor.

Even possessing this information in its knowledge base is, while necessary, not sufficient for the computer to identify it from the text as necessary-for that, the computer should be able to calculate the complex property of saliency, which highlights certain element of meaning as essential for this particular moment in text or information processing and does it highly dynamically (Taylor et al., 2010; Giora, 2003). Saliency stems, of course, from the fact that any natural language statement describes reality very selectively, by highly undermining it (Barwise \& Perry, 1983; Nirenburg \& Raskin, 2004).

The computational analysis of a whole aggregation of jokes and their elicited responses may reveal not only the range of opinion about a particular event, but also indicate, by the absence of humorous postings, the events exempted from humor, thus indicating public indifference. Contrary to one's intuition, and often moral values, jokes about major tragedies appear within days after the events, but they do not typically indicate the values of the joke tellers but rather their reaction to the reporting excesses (Davies, 1998; cf. Oring, 1987; Kuipers, 2005): “What is the best detergent in Indonesia? -Tide.” Thus, they are more of an acknowledgement of occurrence than an indicator of anything else.

Unlike aggregated information analysis, where jokes' usage can be used to indicate public awareness of events, their opinion of them, as well their sensitivity to them (unless they are disaster jokes), the analysis of joke choices of a certain individual can reveal their preferences and opinion as well, all concentrated in the middle section of the joke perception range cleared of its extremes. In other words, the lack of jokes about particular topics indicates either the individual's total indifference to it or their extreme sensitivity (Freud, 1905; cf. Veatch, 1998). This, in turn, can be used to assess an individual's suitability for targeted advertising, focused marketing, or political campaigns. Additionally, if computers act as human partners in communication, as, for instance, in the case of shut-ins (Wilks, 2005), they should, and in this case, they can acquire the appropriate sensitivities for communicating with a given human user, whether jokes are used or not.

There is also the cyber security aspect to individual humor profiling with regard to detecting and protecting against social engineering. Social engineering is understood in cyber security not in its original sense of designing, developing, and modifying social institutions and practices but rather in the sense of a computer criminal posing as somebody he or she is not, to gain access to a network that they are not authorized to use (Raskin et al., 2010b-cf.; Long et al., 2008; Mitnick \& Simon, 2005) for instance, by pretending to be a network technician who needs to fix a problem, to coax a password from s helpful secretary. So, if somebody's individual humor preferences are established and the impostor deviates from them, that should raise a flag. Long shot? Perhaps, but every little bit helps!

\section{Can Machines Understand Humor}

\subsection{Ontological semantic technology}

Taylor (Taylor, 2010) uses Ontological Semantic Technology (OST) to represent the meaning of text in jokes. At the core of OST (Raskin et al., 2009) are repositories of world and linguistic knowledge, acquired semi-automatically within the approach and used to disambiguate the different meanings of 
words and sentences and to represent them comprehensively. These repositories, also known as the static knowledge resources, consist of the ontology, containing language-independent concepts and relationships between them; one lexicon per supported natural language (such as English), containing word senses anchored in the language-independent ontology which is used to represent their meaning; and the Onomasticon, the proper name lexicon, which contains names of people, countries, organizations, etc., and their descriptions, also anchoring them in ontological concepts and interlinking them with other Onomasticon entries. The lexicon and ontology are used by the semantic analyzer, a software that produces Text Meaning Representations (TMRs) from the text that it reads. The format of TMRs conforms to the format and interpretation of the ontology. The processed TMRs are entered into the Information Repository, a dynamic knowledge resource of OST, from which information is used for further processing and reasoning.

The jokes are recognized according to the ontological knowledge of the world. The ontology is constructed with the purpose of handling natural language information, and thus, must accommodate vagueness and fuzziness of natural language. The ontology is governed by the following rules for any concept C, D and relationship R, outlined in Taylor \& Raskin (Taylor \& Raskin, 2010):

$I[C D](x)=\max \{I[C](x), I[D](x)\}$

$I[$ and $C D](x)=\min \{I[C](x), I[D](x)\}$

$I[(\operatorname{Rel}(D))](x)=\max _{y \in D}\{I[\operatorname{Rel}](y, x)\}$

$I[(\operatorname{Rel}($ and $C D))](x)=\min \{I[\operatorname{Rel}(C)](x), I[\operatorname{Rel}(D)](x)\}$

$I[\operatorname{Rel}(C D)](x)=\max \{I[\operatorname{Rel}(C)](x), I[\operatorname{Rel}(D)](x)\}$

$I[C(\operatorname{Rel}(D))](x)=\min \{I[C](x), I[\operatorname{Rel}(D)](x)\}$

$I\left[C\left(\operatorname{Rel}_{1}(D)\right)\left(\operatorname{Rel}_{2}(E)\right)\right](x)$ $=\min \left\{I\left[C\left(\operatorname{Rel}_{1}(D)\right)\right](x), I\left[C\left(\operatorname{Rel}_{2}(E)\right)\right](x)\right\}$

For the construction of the ontology, subsumption definition of Yen (Yen, 1991) was followed: $C$ subsumes $D$ if $\forall x \in D$ : $I[C](x) \geq I[D](x)$.

\subsection{Statistics/machine learning}

Computational humor detection has been attempted through other approaches. The Knock Knock joke recognizer (Taylor \& Mazlack, 2004) was one of the first approaches to humor recognition, limited to a very narrow domain. The approach used n-grams for joke recognition. The One-Liner recognizer (Mihalcea \& Strapparava, 2005; 2006) used machine learning techniques for humor recognition of a slightly wider domain. The authors define a one-liner as "a short sentence with comic effects and an interesting linguistic structure: simple syntax, deliberate use of rhetoric devices (e.g. alliteration, rhyme), and frequent use of creative language constructions meant to attract the reader's attention." A similar approach to one-liners was taken by Mihalcea \& Pulman (Mihalcea \& Pulman, 2007). The authors investigated humorous news articles from The Onion and non-humorous news articles from The Los Angeles Times, the Foreign Broadcast Information Service, and the British National Corpus. Naïve Bayes and Support Vector Machine algorithms were used for text classification. A meaning-based classification of text into humorous and non-humorous using description logic was described in Taylor (Taylor, 2008). Short jokes, without specific form restriction, were selected from joke books and compared to synthesized non-jokes very similar to the selected joke, with the difference that there was no humor in them. All approaches demonstrated successful results.

\subsection{Theory or corpus}

Roughly, there are two approaches to humor detection: one that takes theories and observations derived and described by humans and inserts it into a machine, and the second one that takes a corpus of 
jokes and a corpus of non-jokes and tasks a machine to find differentiating features without using humorrelated human knowledge.

Computationally, there is a significant difference between these approaches. The former is essentialist and representational while the other is corpus-based and depends on the computer ability to capture patterns of distribution that humans do not discern, at least consciously. Both have a purview and a methodology, and both bring about interesting results. Philosophically, one may argue for both positions: on the one hand, one can start with apriori hypotheses about the nature of things and then test them against relevant material; on the other, one can use increasingly advanced techniques to deduce knowledge from data. Both have their own pros and cons, strengths and vulnerabilities, and it is not necessary to review them here, especially since our goal is collaboration, not confrontation. One practical difference pertaining to this paper is that, within this approach, we acquire the semantic resources while our colleagues consider this onerous effort a bottleneck that should be avoided.

We believe that the machine learning approach should be used when we do not have the knowledge for representational resources and rules based on that knowledge. We find this to be the optimal division of labor between the approaches: our OST or any other computational semantic approach, where we have the requisite knowledge to proceed with the rule-based methodology; ML beyond that. This is why, like Minsky (Minsky, 2007), we believe that Mihalcea's and her co-authors' interesting and popular work (Mihalcea \& Pulman, 2007; Strapparava et al., 2011) would yield even more significant results when applied to a much more enriched knowledge base-for instance, when we complete ours. For the purposes of this paper, we may need to customize the best available statistical algorithms for determining individual humor profiles and their aggregation-at least until we acquire advanced knowledge of these phenomena

The importance of understanding the text of jokes, in addition to just detecting them, cannot be underestimated, no matter how it is achieved. Once the joke is detected, understood, and its main parameters such as Target, Situation, Scripts are identified, the real work begins. The opinion indicators, for instance posted comments, are analyzed with a meaning-access technology, such as OST, and juxtaposed on the joke as the reference point. The opinions may introduce additional, related Targets and Situations that can be used in the opinion spectrum analysis, organized by the identified Targets and Situations in both jokes and their non-joke responses. It should be noted that Targets and Situations can be used as the single axis each or as specific pairs.

At the same time, all analyzed jokes used or commented on by a specific individual can be processed to identify his or her humor preferences and related social opinions, attitudes and positions. Such positions can be analyzed for any one period of time, or longitudinally over any desired period of time for which the material is available. Unlike the aggregated analysis, where information is processed per instance of a parameter or a pair of instances, for the individual analysis, the obtained information has to be combined together to build an overall picture from which inferences can be made. It goes without saying that information learned about a particular individual can be used to model companion-type communication with a computer, as the computer now possesses valuable information about the person's preferences and sensitivities not just in the read jokes but based on the overall picture that was calculated for this individual. 


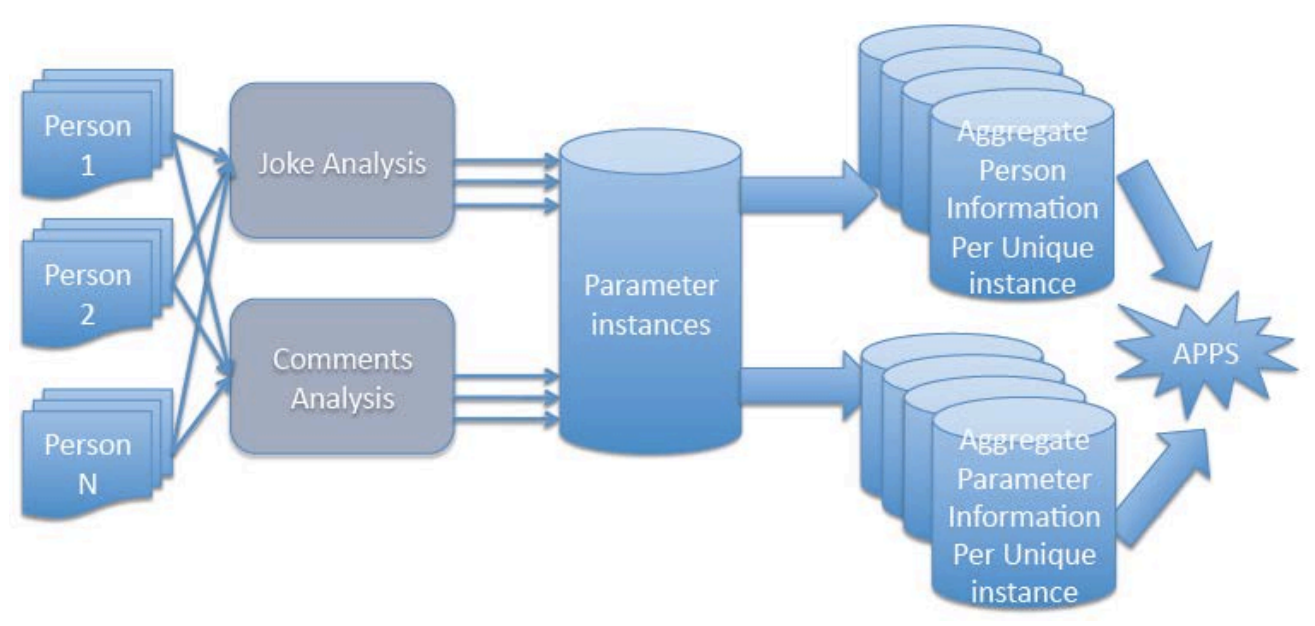

Fig. 5. Processing Flow.

Thus processed (Figure 5), both the aggregated and individual data can be fed into any application, in addition and in conjunction with the state of the art technology. It can be anticipated that the set of current candidates for applications using this research will continue to grow rapidly with time. For instance, in the light of the increasing interest in social computing (Morkes et al., 1998; Charron et al., 2006) and hybrid agent-human communication systems (Matson et al., 2011 and references there), the role of humor can be expected to increase significantly as such systems will attempt to emulate the natural flow of conversations among the humans where humor has always occupied significant space.

\section{Conclusion}

In this paper, we have outlined the nature of a proper formal and computational theory of humor and then addressed the theoretical framework for and methodological approach to computing human attitudes and opinions by analyzing the humor they use and/or comment on. The theoretical framework consists of two major components, namely, a formal theory of humor and a meaning-based theory of computational semantics. In our methodological approach, both components are based on Ontological Semantic Technology. Since the information that we process is perception-based, we employ fuzzy logic in the text processing component, that was designed to deal with these perceptions. We would like to emphasize also that the vagueness and fuzziness components of the system will need to be much further developed for the applications to be truly successful. Parts of the proposed system have already been implemented, both in terms of semantic interpretation of text and in computing humor. Future research will lead us to the implementation of a smallish-scale pilot system and then on to a practical industrial application, which is our ultimate goal, both for the purposes of a practical achievement and of the empirical justification and validation of the theory and methodology.

\section{References}

Apte, M. (1988). Disciplinary Boundaries in Humorology: An Anthropologist's Ruminations. Humor: International Journal of Humor Research, 1(1), 5-25.

Attardo, S. (1994). Linguistic Theories of Humor: Mouton de Gruyter.

Attardo, S., Hempelmann, C. F. \& Di Maio, S. (2002). Script Oppositions and Logical Mechanisms: Modeling Incongruities and Their Resolutions. Humor: International Journal of Humor Research, 15(1), 1-44.

Attardo, S., \& Raskin, V. (1991). Script Theory Revis(it)ed: Joke Similarity and Joke Representation Model. Humor: International Journal of Humor Research, 4 (3-4). 
Barwise, J., \& Perry, J. (1983). Situations and Attitudes: MIT Press-Bradford.

Carrell, A. T. (1993). Audience/Community, Situation, and Language: A Linguistic/Rhetorical Theory of Verbal Humor, Unpublished Ph.D. Dissertation, Department of English, Purdue University, W. Lafayette, IN.

Carrell, A. T. (1997a). Humor Communities. Humor: International Journal of Humor Research, 10(1), 11-24.

Carrell, A. T. (1997b). Joke Competence and Humor Competence. Humor: International Journal of Humor Research, 10(2), 173-185.

Carrell, A. T. (2008). Historical Views of Humor. In V. Raskin (ed.), The Primer of Humor Research: Mouton de Guyter.

Charron, C., Favier, J. \& Li, C. (2006). Social Computing: How Networks Erode Institutional Power, And What to Do About It: Forrester Research, Forrester.com.

Chomsky, N. (1965). Aspects of the Theory of Syntax: MIT Press.

Davies, C. (1998). Jokes and Their Relations to Society: Mouton de Gruyter.

Davies, C. (2011). Logical Mechanisms: A Critique. Humor: International Journal of Humor Research, 24(2), 159-166.

Deneire, M. (1995). Humor and Foreign Language Teaching. Humor: International Journal of Humor Research, 8(3), 286-298.

Ferrucci, D., Brown, E., Chu-Carroll, J., Fan, J., Gondek, D., Kalyanpur, A. A., Lally, A., Murdock, W., Nyberg, E., Praeger, J., Schlaefer, N., and Welty, C. (2010). Building Watson: An Overview of the DEEPQA Project. AAAI Magazine, Fall.

Giora, R. (2003). On Our Mind: Salience, Context, and Figurative Language: Oxford University Press.

Gruner, C. R. (1978). Understanding Laughter: The Workings of Wit and Humor: Nelson-Hall.

Hempelmann, C. F. (2004). Script Opposition and Logical Mechanism in Punning. Humor: International Journal of Humor Research, 17(4), 381-392.

Hempelmann, C. F. (2009). Pseudo-Logical Mechanism: Failed Artificial Intelligence. Presentation at ISHS '09: Annual Meeting of the International Society of Humor Studies, Long Beach, CA: California State University.

Hempelmann, C. F. (2011). Explicitly Is Proverbially and at This Point in This Title Also Obviously the Death of Wit, or Is It? Paper presented at ISHS '11: Annual Meeting of the International Society of Humor Studies, Boston, MA: Boston University.

Hempelmann, C. F. (2012). The Falsity of Fun. Paper presented at ISHS '12: Annual Conference of the International Society of Humor Studies, Cracow, Poland: Jagellonian University.

Hempelmann, C. F. \& Attardo, S. (2011). Resolutions and Their Incongruities: Further Thoughts on Logical Mechanisms, Humor: International Journal of Humor Research, 24(2). 125-149.

Kant, I. (1790). Kritik der Urteilskraft: Lagarde. Engl. transl.: (1951). Critique of Judgment: Hafner.

Kuipers, G. (2002). A Hackle of V. Raskin’s Plenary, ISHS 2002, Bertinoro, Italy.

Kuipers, G. (2005). “Where Was King Kong When We Needed Him?’’ Public Discourse, Digital Disaster Jokes, and the Functions of Laughter after 9/11. The Journal of American Culture, 28:1.

Long, J., Wiles, J., Pinzon, S., and Mitnick, K. D. (2008). No Tech Hacking: A Guide to Social Engineering, Dumpster Diving, and Shoulder Surfing: Syngress.

Martin, R. A. (2008). Humor and Health. In V. Raskin (ed.), The Primer of Humor Research: Mouton de Gruyter (479-522).

Matson, E., Taylor, J., Raskin, V., Min, B.-C. \& Wilson, E. (2011). A Natural Language Model for Enabling Human, Agent, Robot and Machine Interaction. The 5th IEEE International Conference on Automation, Robotics and Applications, Wellington, New Zealand.

Mihalcea, R., \& Pulman, S. (2007). Characterizing Humour: An exploration of Features in Humorous Texts. Springer Lecture Notes in Computer Science 4394, 337-347.

Mihalcea, R., \& Strapparava, C. (2005). Computatinal Laughing: Automatic Recognition of Humorous One-Liners. Proceedings of Cognitive Science Conference, 1513-1518. 
Mihalcea, R., \& Strapparava, C. (2006). Learning to Laugh (Automatically): Computational Models for Humor Recognition. Computational Intelligence, 22 (2), 126-142.

Mihalcea, R., Strapparava, C., \& Pulman, S. (2010). Computational Models for Incongruity Detection in humor. Proc.of CICLing 2010: Conference on Computational Linguistics and Intelligent Text Processing, Iasi, Romania.

Mitnick, K. D., \& Simon W. L. (2005). The Art of Intrusion: The Real Stories Behind the Exploits of Hackers, Intruders and Deceivers: Wiley.

Minsky, M. (2007). Emotion Machine: Commonsense Thinking, Artificial Intelligence, and the Future of the Human Mind. Presentation at MIT World: http://mitworld.mit.edu/video/484

Morkes, J., Kernal, H. K., \& Nass, C. (1998). Humor in task-oriented computer-mediated communication and human-computer interaction. Proceedings of CHI: ACM.

Morreall, J. (1983). Taking Humor Seriously: SUNY Press.

Nirenburg, S., \& Raskin, V. (2004). Ontological Semantics: MIT Press.

Oring, E. (1987). Jokes and the Discourse on Disaster. The Journal of American Folklore, 100: 397, 276286.

Oring, E. (1992). Jokes and Their Relations: University Press of Kentucky.

Oring, E.: Parsing the Joke: The General Theory of Verbal Humor and Appropriate Incongruty, Humor: International Journal of Humor Research, 24(2), 203-222.

Rapp, A. (1951). The Origins of Wit and Humor: Dutton.

Raskin, V. (1985). Semantic Mechanisms of Humor: D. Reidel.

Raskin, V. (2011a). Length and Girth Saliency in Jokes. Paper presented at ISHS '11: Annual Meeting of the International Society of Humor Studies, Boston, MA: Boston University.

Raskin, V. (2011b). On Oring on GTVH, Humor: International Journal of Humor Research, 24(2), 223232.

Raskin, V., Hempelmann, C. F., \& Taylor, J. M. (2009a). From SSTH to GTVH to OSTH-and never ever back! 2009 Annual Meeting of the International Society for Humor Studies-ISHS'09: International Conference on Humor Research, Long Beach, CA: University of California.

Raskin, V., Hempelmann, C. F., \& Taylor, J. M. (2009b). How to Understand and Assess a Theory: The Evolution of the SSTH into the GTVH and Now into the OSTH, Journal of Literary Theory.

Raskin, V., Hempelmann, C. F., \& Taylor, J. M. (2010a). Guessing vs. Knowing: The Two Approaches to Semantics in Natural Language Processing, Annual International Conference Dialogue 2010, Moscow, Russia.

Raskin, V., Taylor, J. M., \& Hempelmann, C. F. (2011). Ontological Semantic Technology for Detecting Insider Threat and Social Engineering, Pre-Proc. NSPW-10, Concord, MA, 2010b. Reprinted in K. Beznosov (ed.), NSPW-10: Proceedings of the 2010 New Security Paradigms Workshop, Concorde, MA, September 20-23: ACM Press, 2011.

Raskin, V., Nirenburg, S., Hempelmann, C. F., Nirenburg, I., \& Triezenberg, K. E.: The Genesis of a Script for Bankruptcy in Ontological Semantics. In: G. Hirst and S. Nirenburg (eds.), Proceedings of the Workshop on Text Meaning, 2003 NAACL Human Language Technology Conference, 27-31.

Ritchie, G. (2000). Describing Verbally Expressed Humour. Proceedings of AISB Symposium on Creative and Cultural Aspects and Applications of AI and Cognitive Science, 71-78. Birmingham, UK.

Ritchie, G. (2004). The Linguistic Analysis of Jokes: Routledge.

Ruch, W. (1998). The Sense of Humor: Mouton de Gruyter.

Ruch, W., Attardo, S, \& Raskin, V. (2003).Toward an Empirical Verification of the General Theory of Verbal Humor. Humor: International Jornal of Humor Research, 6 (2), 123-136.

Strapparava, C., Stock, O., \& Mihalcea, R. (2011). Computational humour. In R. Cowie, P. Petta, and C. Pelachaud (eds.), Emotion-Oriented Systems: The HUMAINE Handbook (Cognitive Technologies, Chapter 6.4): Springer.

Suls, J. (1976). Cognitive and Disparagement Theories of Humour: A Theoretical And Empirical Synthesis. In A. J. Chapman, \& H. C. Foot (eds.), It's a Funny Thing Humour: Pergamon Press. 
Taylor, J. M. (2008). Towards Informal Computer Human Communication: Detecting Humor in Restricted Domain, Unpublished Ph.D. Dissertation, Department of Computer Science, University of Cincinnati.

Taylor, J. M. (2010). Ontology-Based View of Natural Language Meaning: The Case of Humor Detection, Journal of Ambient Intelligence and Humanized Computing, 1:3, 221-234.

Taylor, J. M. (2011). Does SO2 Always Result in a Joke: How Long Is Long enough? Paper presented at ISHS '11: Annual Meeting of the International Society of Humor Studies, Boston, MA: Boston University.

\section{Author Biographies}

Julia M. Taylor (Ph.D. in Computer Science and Engineering, University of Cincinnati, USA, 2008) implemented the first ever computational meaning-based verbal-joke detector as in her dissertation. Before and after that, she has contributed significantly to the development of the formal theoretical and computational linguistic theory of humor, most recently having co-founded the Ontological Semantic Theory of Humor. After a few years in natural language processing industry, developing and implementing the Ontological Semantic Technology, she is an Assistant Professor of Computer and Information Technology at Purdue University, West Lafayette, Indiana, USA. Dr. Taylor has published several dozen papers in computational semantics, artificial intelligence, robotic communication, and, of course, humor research. An SDPS participant since 2011 and a session co-organizer and presenter at SDPS 2012, she has been an active member of the Next Generation Leadership Team.

Victor Raskin (Ph.D. in Mathematical, Theoretical and Computational Linguistics, Moscow State University, USSR, 1970) is a co-founder of computational semantics in the late 1960s-early 1970s and a founder of the first meaning-based theory of verbal humor (Script-based Semantic theory of Humor-SSTH). He has been also a co-founder of its successors, the Generative Theory of Verbal Humor (GTVH) and, lately, the Ontological Semantic Theory of Humor (OSTH). The author, co-author, or editor of some two dozen books and over 200 papers in theoretical and computational semantics, philosophy of language, artificial intelligence, robotic intelligence and communication, and humor research, Dr. Raskin is Distinguished Professor of English and Linguistics and, by courtesy appointments, Professor of Computer Science and of Computer and Information Technology at Purdue University, West Lafayette, Indiana, USA. He was also an invited plenary speaker at SDPS 2011 as well as a session co-organizer and presenter at SDPS 2012. 